In der Rubrik „Literatur kompakt" werden die wichtigsten Originalarbeiten aus der internationalen Fachliteratur referiert.

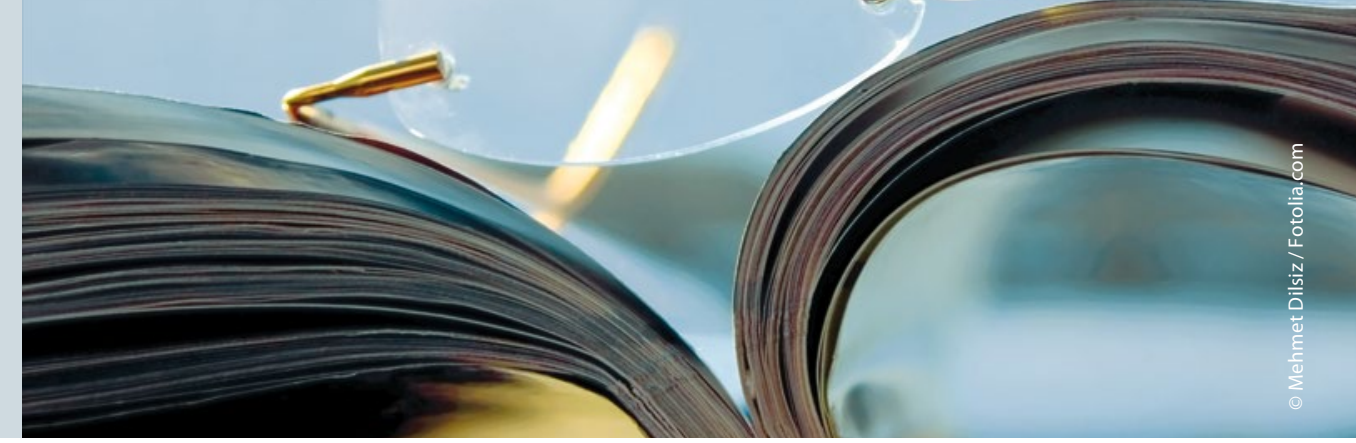

\title{
GLP-1-Agonist verbessert Schlafapnoe bei adipösen Patienten
}

\begin{abstract}
Mit zunehmendem Körpergewicht steigt das Risiko für eine Schlafapnoe. Der GLP-1-Agonist Liraglutid konnte in einer Studie nicht nur Blutzucker und Blutdruck reduzieren, sondern auch Gewicht und Schlafapnoe.
\end{abstract}

In die Untersuchung wurden 359 Personen mit einem Alter von 18-64 Jahren und einem $\mathrm{BMI}>30 \mathrm{~kg} / \mathrm{m}^{2}$ aufgenommen, aber keine Diabetiker. Alle Teilnehmer hatten eine mittel- oder hochgradige obstruktive Schlafapnoe (15-29,9 bzw. > 30 Ereignisse/ Stunde). Je die Hälfte von ihnen wurde mit dem GLP-1-Agonisten Liraglutid (täglich 3,0

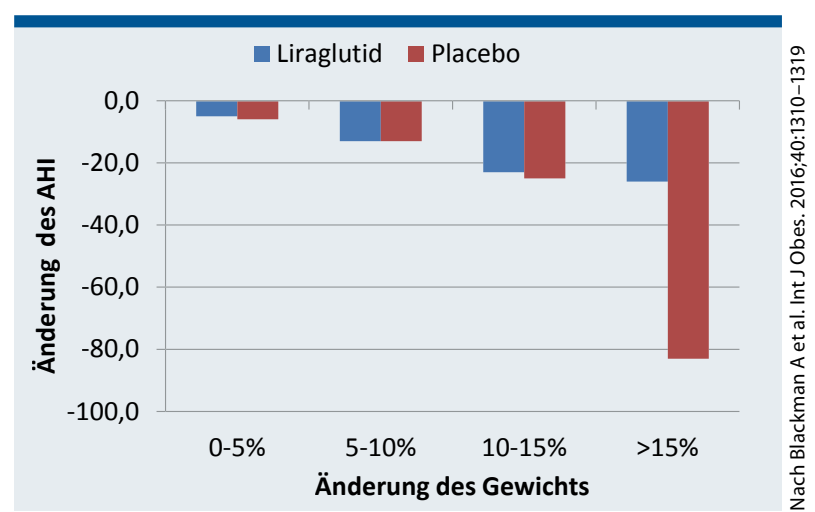

Abb. 1: Änderung des Apnoe-Hypopnoe-Index (AHI) bei Gewichtsabnahme. mg) oder Placebo behandelt. Allen Teilnehmern wurde eine Reduktionskost mit einem Energiedefizit von $500 \mathrm{kcal} / \mathrm{Tag}$ sowie mehr Bewegung empfohlen.

Nach 32 Wochen war die Gewichtsabnahme unter Liraglutid mehrfach höher als unter Placebo (-6,7 vs. $-1,9 \mathrm{~kg}$ ) und die Reduktion des Apnoe-Hypopnoe-Index (AHI) doppelt so stark ausgeprägt wie unter Placebo (-12,2 vs. $-6,1$ Ereignisse/Stunde). Die Kriterien einer obstruktiven Schlafapnoe (< 5 Ereignisse/ Stunde) waren bei 5,4 vs. $1,2 \%$ der Patienten nicht mehr vorhanden. Die Verbesserung der Apnoe unter Liraglutid war umso deutlicher, je gravierender die Schlafapnoe und je stärker ausgeprägt die Gewichtsabnahme war (Abb. 1). Die Sau- erstoffsättigung, die Schlafzeit und Parameter zur Lebensqualität verbesserten sich in der Tendenz. Der systolische Blutdruck sank ( $-3,4$ vs. $0,0 \mathrm{mmHg})$, und die Umfänge von Bauch $(-6,4$ vs. $3,1 \mathrm{~cm})$ und Nacken (-2,2 vs. $-1,3 \mathrm{~cm})$ nahmen ab.

Blackman A et al. Effect of Liraglutide $3,0 \mathrm{mg}$ in individuals with obesity and moderate or severe obstructive sleep apnea: the SCALE Sleep Apnea randomized clinical trial. Int J Obes. 2016; 40:1310-1319.

\section{Kommentar}

Eine Schlafapnoe liegt bei etwa 15\% der Männer und $5 \%$ der Frauen im mittleren Alter vor. Zwei Drittel von ihnen haben zu viel Gewicht, ca. $60 \%$ der Schlafapnoefälle sind durch Überwicht und Adipositas verursacht.
Die obstruktive Schlafapnoe ist eine ernst zu nehmende Krankheit, da sie nicht nur tagsüber mit Müdigkeit und Schläfrigkeit einhergeht und ein erhöhtes Unfallrisiko bedingt, sondern auch durch Begleit- und Folgekrankheiten die Lebenserwartung vermindert.

Die Standardtherapie, die nächtliche Beatmung, vermindert effektiv die Beschwerden, sie wird jedoch nicht von allen Patienten toleriert und ändert auch nicht die häufige Ursache Adipositas. Viele Studien zeigen, dass eine Gewichtsreduktion die obstruktive Schlafapnoe günstig beeinflusst, Liraglutid verstärkt diesen Effekt; Liraglutid ist nicht nur als Antidiabetikum, sondern seit 2015 auch zur Gewichtsreduktion in Deutschland zugelassen.

Um Symptome der obstruktiven Schlafapnoe gänzlich zu beseitigen, ist meist eine Gewichtsabnahme von $>15 \%$ des Ausgangsgewichts anzustreben, was am besten durch eine mehrmonatige Formuladiät oder eine bariatrische Operation gelingt.

Die der Adipositas zugrundeliegenden Mechanismen hinsichtlich der Entwicklung einer obstruktiven Schlafapnoe bzw. einer Gewichtsreduktion sind nur teilweise bekannt. Vermehrte Fettansammlungen im Rachenraum und Nacken sowie im Bauchraum werden als Ursache angenommen.

Fazit: Eine Gewichtsreduktion durch Lebensstiländerung allein oder in Kombination mit Liraglutid verbessert bzw. beseitigt die obstruktive Schlafapnoe bei Adipösen. Ähnlich dürfte das auch bei Diabetikern sein, die in dieser Studie jedoch ausgeschlossen waren.

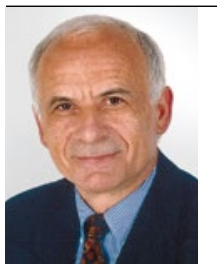

Dr. med. Alfred Wirth Bad Rothenfelde 